\title{
Oxytocin treatment in pediatric populations
}

\author{
Adrienne E. Taylor ${ }^{1 *}$, Hsu-en Lee ${ }^{1,2}$ and Femke T. A. Buisman-Pijlman ${ }^{3}$ \\ ${ }^{1}$ Department of Psychological Medicine, Women's and Children's Hospital, Adelaide, SA, Australia \\ 2 School of Medicine, The University of Adelaide, Adelaide, SA, Australia \\ ${ }^{3}$ Discipline of Pharmacology, Faculty of Health Sciences, School of Medical Sciences, The University of Adelaide, Adelaide, SA, Australia
}

\section{Edited by:}

Lisa A. Parr, Emory University, USA

Reviewed by:

Valery Grinevich, German Cancer

Research Center DKFZ and

University of Heidelberg, Germany

Bernadette Von Dawans, University

of Freiburg, Germany

*Correspondence:

Adrienne E. Taylor, Department of

Psychological Medicine, Women's

and Children's Hospital, 72 King

William Road, North Adelaide,

Adelaide, SA 5006, Australia

e-mail: adrienne.taylor@

health.sa.gov.au
The role of endogenous oxytocin as neuromodulator of birth, lactation and social behaviors is well-recognized. Moreover, the use of oxytocin as a facilitator of social and other behaviors is becoming more and more accepted. Many positive effects have been attributed to intranasal oxytocin administration in animals and humans; with current research highlighting encouraging advances in its potential for use in mental health disorders. The new frontier will be investigating the effective use of oxytocin in pediatric populations. Limited animal data is available on this. Large-scale human studies focusing on autism are currently under way, but many other possibilities seem to lie in the future. However, we need to know more about the risks and effects of repeated use on the developing brain and body. This paper will provide an overview of the current understanding of the role of endogenous oxytocin and its related neuropeptide systems in influencing behaviors, in particular attachment, and will review (a) the literature on the use of intranasal oxytocin in young animals, children (age range birth-12 years) and adolescents (age range 13-19 years), (b) the expected benefits and risks based on the current research, and (c) the risks of oxytocin in children with severe psychopathology and early life trauma. The paper will conclude with a clinical perspective on these findings.

Keywords: oxytocin, neuropeptide, attachment, pediatric populations, intranasal oxytocin

\section{INTRODUCTION}

Current understanding of oxytocin and its related neuropeptide systems is growing at an exponential rate. Oxytocin's centrally acting role in behavioral modulation and attachment is increasingly recognized (Feldman, 2012; Churchland and Winkielman, 2012; Tops et al., 2014). Recent studies (Broadbear et al., 2014; Love, 2014; Tops et al., 2014) have been able to highlight a neurobiological model for these behavioral changes, and outline with greater specificity the complex interaction between oxytocin and other neuropeptides and monoamines. These findings have been applied and expanded to the field of addiction, and behavioral neuroscience (Kovacs et al., 1998; Rilling et al., 2012; Buisman-Pijlman et al., 2014b; Williams and Johns, 2014; Zanos et al., 2014). Many positive effects have now been attributed to intranasal oxytocin in the adult population (Veening and Olivier, 2013), however there are multiple questions that remain unanswered in regards to the safe and effective use of intranasal oxytocin in the child and adolescent population. In particular, there is a lack of evidence in regards to the effects of both acute and chronic exposure on the developing brain. This paper aims to provide a review of current knowledge in relation to this area, and highlight both theoretical and clinical gaps in research evidence and data regarding what will likely be an increasing area of interest in the management of common childhood and adolescent psychiatric disorders in future.

This paper will briefly review the current understanding of the role of endogenous oxytocin and its related neuropeptide systems in influencing behaviors, in particular attachment, and will review (a) the literature on the use of intranasal oxytocin in young animals, infants, children and adolescents, (b) the expected benefits and risks based on the current research, and (c) the risks of oxytocin in children with severe psychopathology and early life trauma. Finally this paper aims to utilize the current knowledge base to assist in providing a framework for the direction of potential further clinical application and will provide examples for future research questions.

\section{OXYTOCIN AND ITS RELATED NEUROPEPTIDE SYSTEMS}

Oxytocin, a hormone synthesized by the hypothalamus and subsequently secreted by the posterior pituitary gland, is a neuropeptide well-known for its neuro-modulatory role in lactation, child-birth, mother-infant bonding (Love, 2014), and more recently for its role in social affiliation and partner attachment in adults (Buisman-Pijlman et al., 2014b; Tops et al., 2014). Oxytocin's centrally acting effects form the basis of research stemming from Nobel laureate in Chemistry Vincent du Vigneaud's early descriptions and syntheses of oxytocin and vasopressin in 1953 (Love, 2014; Sarnyai and Kovacs, 2014). Oxytocin has it's effect through the Oxytocin receptor and Vasopressin 1A Receptor. Immunohistochemical studies have revealed that magnocellular oxytocin neurons of paraventricular, supraoptic, and accessory hypothalamic nuclei via local dendritic and distant axonal release of oxytocin modulate various forms of behaviors (Lee et al., 2009; Knobloch et al., 2012; Dolen et al., 2013). It is possible that axonal projections of oxytocin neurons can be altered in patients afflicted with neurodevelopmental diseases, 
which may impair oxytocin signaling and contribute to defects in social behavior. Such pathophysiological mechanisms require further research.

Oxytocin has been shown to have a relationship with both vasopressin and serotonergic neural pathways (Broadbear et al., 2014), with increasing evidence suggesting that oxytocin and vasopressin's individual and combined modulatory influences on related neurotransmitter systems, including dopaminergic, serotonergic, and endogenous opioid system play a crucial role in the brain's motivational and reward pathways (Love, 2014), and behaviors related to stress-responses and addiction (BuismanPijlman et al., 2014b; Tops et al., 2014). Oxytocin has also been implicated in modulating the reactivity of the hypothalamicpituitary-adrenal axis (Buisman-Pijlman et al., 2014b), thus in part determining the extent of psychological and physiological response to stress (Feldman, 2012).

This expanding knowledge base has underpinned adult human and animal studies administrating intranasal oxytocin as a modulator of behaviors, for example, those associated with substance abuse (McGregor and Bowen, 2012; Pedersen et al., 2013). Authors have outlined the rationale for intranasal administration of oxytocin in research, based on this administration route bypassing the blood-brain barrier, and increasing the half-life of the delivered substance in cerebrospinal fluid thus ensuring maximization of centrally acting effects (Veening and Olivier, 2013). Intranasal delivery accesses rostral and possibly other brain areas following absorption through the nasal epithelium (Veening and Olivier, 2013). In the pediatric population, intranasal delivery of oxytocin is likely to be the easiest, least intrusive and most preferable mode of administration given pragmatic factors relating to level of insight into treatment, and aversion behaviors, however this needs further investigation.

From a clinical perspective, Intellectual Disabilities, Autism Spectrum Disorders (ASDs) and ADHD are common neurodevelopmental disorders presenting in childhood. Whilst neurodevelopmental disorders frequently require ongoing treatment and management in adolescent populations, this is an age group in which affective disorders, anxiety disorders and eating disorders, or traits thereof, also start to manifest and may require clinical intervention. In the child and adolescent this cohort, disorders are frequently and universally, however not exclusively, underpinned by a developmental history characterized by attachment difficulties or early life trauma.

\section{OXYTOCIN'S ROLE IN ATTACHMENT}

An important precursor to the investigation of oxytocin's role within pediatric populations is the concept of attachment. From a theoretical perspective, Bowlby defines attachment as "a biological instinct...Attachment behavior anticipates a response by the attachment figure which will remove threat or discomfort" (Bowlby, 1960). Oxytocin has been implicated in priming the bond between a mother and child (Love, 2014), and it is known from animal studies that the development of the oxytocin system continues throughout the postnatal period in an experiencedependent manner (Oreland et al., 2010). Given that development of secure attachment is in part reliant on an infant's salience toward their primary caregiver, evidence that administration of oxytocin improves the salience of social cues further highlights the importance of oxytocin in the process of attachment (Love, 2014; Tops et al., 2014).

\section{VARIATIONS IN THE OXYTOCIN SYSTEM}

Notably, findings from animal and human studies display that there are individual variations within basal oxytocin levels and in reactivity of the system. Whilst genetics contribute in part, environmental influences such as social experiences, stress and trauma early in life have also been shown to affect this variation. Buisman-Pijlman et al. (2014b) outlined that these influences can induce long-term changes to the oxytocin system, having a subsequent impact on an individual's susceptibility to addiction and relapse. Feldman (2012) also noted that the human oxytocin system is sensitive to both positive early social experiences and negative experiences such as separation. Rather than a static feedback system, it is now more accepted through recent studies that the human oxytocin system functions in a complex context-dependent manner (Feldman, 2012; Guastella and Macleod, 2012). Factors aside from genetic influence that have been implicated in variations in an individual's oxytocin system include prenatal maternal stress and substance use (Johns et al., 1998), early life trauma including parental separation (Opacka-Juffry and Mohiyeddini, 2012), and childhood sexual abuse (Heim et al., 2009). A sound understanding of these factors will be of importance to assess the risks and benefits of exogenous oxytocin in the pediatric population.

\section{CURRENT EVIDENCE ON THE USE OF INTRANASAL OXYTOCIN IN YOUNG ANIMALS, INFANTS, CHILDREN, AND ADOLESCENTS}

In the adult population, literature on the use of intranasal oxytocin has spanned the last six decades in research, with early reports focusing on its role in obstetrics (Hendricks and Pose, 1961; Cohen et al., 1962), followed by exploration of oxytocin's possible application to the field of psychiatry, in particular, obsessive compulsive disorder (Ansseau et al., 1987; Den Boer and Westenberg, 1992) and symptoms of post-traumatic stress disorder (Pitman et al., 1993; Olff, 2012).

In the last 10 years, authors have highlighted oxytocin's prosocial effects in the adult population, categorized further into "trust" (Baumgartner et al., 2008), "mind-reading" and "faceprocessing and memory" (Heinrichs et al., 2003; Beetz et al., 2012) and stress-response in animals (Parker et al., 2005). It is these benefits, in addition to genetic studies displaying a consistent pattern of oxytocin involvement in ASDs (Campbell et al., 2011), that have led to an interest in the promise of treatment of ASDs with intranasal oxytocin. A recent systematic review of randomized control trials of oxytocin intervention in autism by Preti et al., noted that whilst promising findings in the measures of emotion recognition and eye gaze, there is need for better powered studies prior to valid clinical recommendations being made (Preti et al., 2014).

Prior to recent years, animal studies relating to the effects of oxytocin have mainly focused on single dose administration, and in an adult population. On review of current animal data (Table 1), there were four studies identified investigating 


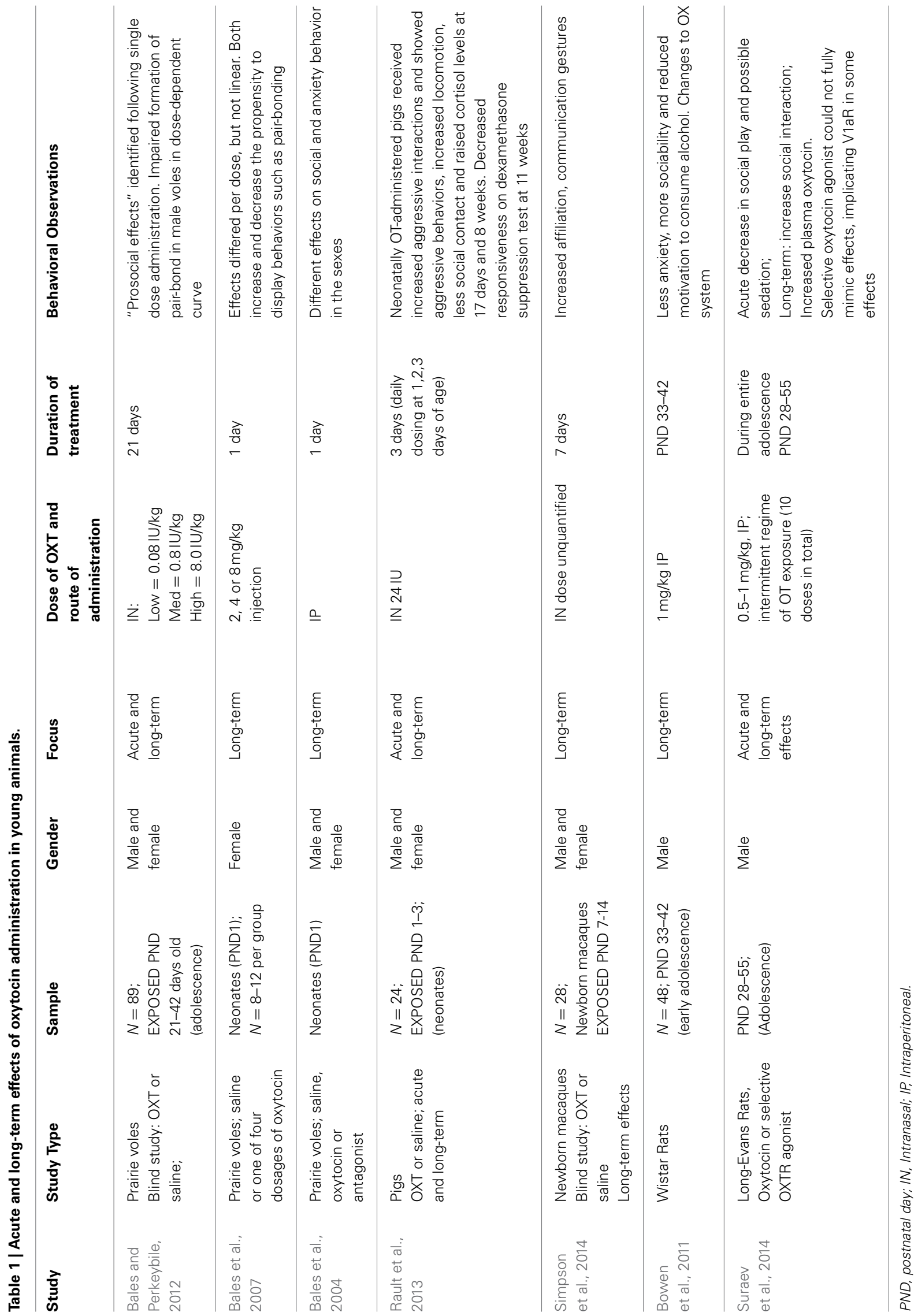


administration of oxytocin via intraperitoneal injection, and three studies using intranasal oxytocin in young.

Studies focusing on intraperitoneal oxytocin injection reinforced inconsistent findings displayed in earlier data according to dose and measured outcome variable, and further highlighted that in the short-term, oxytocin administration may lead to a decrease in positive social behaviors (Suraev et al., 2014), whilst in the long term contribute to heightened social interaction and diminished display of anxiety (Bowen et al., 2011) (see Table 1).

All three animal studies investigating intranasal oxytocin involved repeated dosing. Firstly, Bales et al., found repeated dosing during adolescence had long-term effects on partner preference in prairie voles (Bales et al., 2013). Secondly, Rault et al., found that neonatal pigs displayed increased aggressive behaviors and locomotion, and diminished social contact following daily intranasal oxytocin administration in days 1-3 of life. In addition, there was decreased responsiveness on dexamethasone suppression test at 11 weeks, suggesting repeated intranasal oxytocin administration in early life can cause chronic dysregulation of the hypothalamic-pituitary axis (Rault et al., 2013). Most recently, Simpson et al., found that in newborn rhesus monkeys, administration of daily intranasal oxytocin increased an infant's affiliative communication gestures and decreased salivary cortisol levels (Simpson et al., 2014). In a similar trend identified in the administration of oxytocin via intraperitoneal injection in young animals, the use of intranasal oxytocin produced largely variable results, which appeared to be dose-dependent. Whilst prosocial effects and a decrease in salivary cortisol levels were some of the positive outcomes observed, authors also commented on intranasal oxytocin administration in young animals having the potential to increase aggressive behaviors, impair pair-bond formation, and lead to disruption of the HPA-axis.

A thorough review of current literature found that there were only six human studies (Table 2) focusing on the use of intranasal oxytocin in a child or adolescent population. Of these studies, five occurred in populations with a diagnosis of ASDs (Guastella et al., 2010; Gordon et al., 2013; Tachibana et al., 2013; Dadds et al., 2014; Guastella et al., 2014), and one in a population with Fragile X Syndrome (Hall et al., 2012). Of these six studies, Guastella et al.'s (2014) RCT investigating the effects of a course of intranasal oxytocin on social behaviors in youths with ASDs, and Tachibana et al., were the only groups to investigate chronic administration of intranasal oxytocin over 2, and 6 month periods, respectively, although there was notably a small sample size in the later study. Moreover, only three of the relevant studies found on review included children under the age of 12 (Gordon et al.; Dadds et al.; Tachibana et al.), with a clear lack of data pertaining to the effect of intranasal oxytocin administration in the neonatal and early childhood/pre-pubertal stages of development, a time during which the oxytocin system displays its highest degree of neuroplasticity. From a broader perspective, review of the current literature of intranasal oxytocin use in child and adolescent populations highlighted significant limitations to inferring clinically significant results, and recognizing consistent patterns of effect between studies, including: small sample sizes, limited data on chronic use compared to single-dose administration, and a predilection toward male subjects.
Studies identifying beneficial clinical outcomes to intranasal oxytocin administration were (1) Gordon et al., who found improved performance in detecting socially salient information on a well-validated fMRI emotion judgment task ("Reading the Mind in the Eyes Test") following intranasal oxytocin, in addition to identifying that oxytocin enhances activity in social brain regions (see Table 2) (Gordon et al., 2013), (2) Guastella et al., in their 2010 crossover study who displayed improved performance on the RMET in $60 \%$ of subjects following intranasal oxytocin administration (Guastella et al., 2010), (3) Hall et al., who reported improved frequency of eye gaze at a specific dose oxytocin, in addition to decreased salivary cortisol levels in subjects receiving a specific dose (Hall et al., 2012) and (4) Tachibana et al.,'s study revealing six of a total 8 male youths with a primary diagnosis of ASD displayed improved performance on communication and social interaction domains of the Autism Diagnostic Observation Schedule-Generic (ADOS-G) following incremental dose increases of intranasal oxytocin over a 6 month period (see Table 2).

The most recent studies by Dadds et al., and Guastella et al., do not report identified significant clinical benefit following the administration of oxytocin.

Despite an evolving scope of current animal and human research data relating to the use of intranasal oxytocin in adults, studies relevant to young animals, and the pediatric population remain limited to date. Factors contributing to this may be related the uncertainty of risks of single, or multiple oxytocin dose administrations on the developing brain, in addition to an absence of clear methods to measure outcome (Miller et al., 2013). Review highlighted no significant reporting of side effects or adverse events in the studied populations, with multiple studies referring to intranasal oxytocin being well-tolerated.

\section{THE EXPECTED BENEFITS AND RISKS OF INTRANASAL OXYTOCIN IN PEDIATRIC POPULATIONS}

In assessing expected risks and benefits of oxytocin administration in the pediatric population, it will be important to first consider basic principles of neuroadaptation, including the development of tolerance, changes in receptor numbers, and receptor sensitivity, related to repeated exposure of any drug.

Based on current research data in adult animal and human populations, and the promising findings noted in behavioral modulation (Miller, 2013; Love, 2014), translational studies to the pediatric population could too hold promise. There is a potentially broad scope of application for the use of intranasal oxytocin based on mental health disorders or presentations that are characterized by a decreased responsiveness to social cues, emotional dysregulation and aggression. It would also be of interest to determine if the positive findings in the field of oxytocin and substance addiction (Bowen et al., 2011; Buisman-Pijlman et al., 2014a) were reproducible in younger populations.

As outlined by Miller et al. (2013) in their study reviewing the role of gender in regards to symptomatology in ASDs, there are variations in oxytocin and vasopressin identified between sexes (Miller et al., 2013). Broader application of this principle and an understanding of baseline variations in endogenous oxytocin levels between sexes will likely be important in future research 


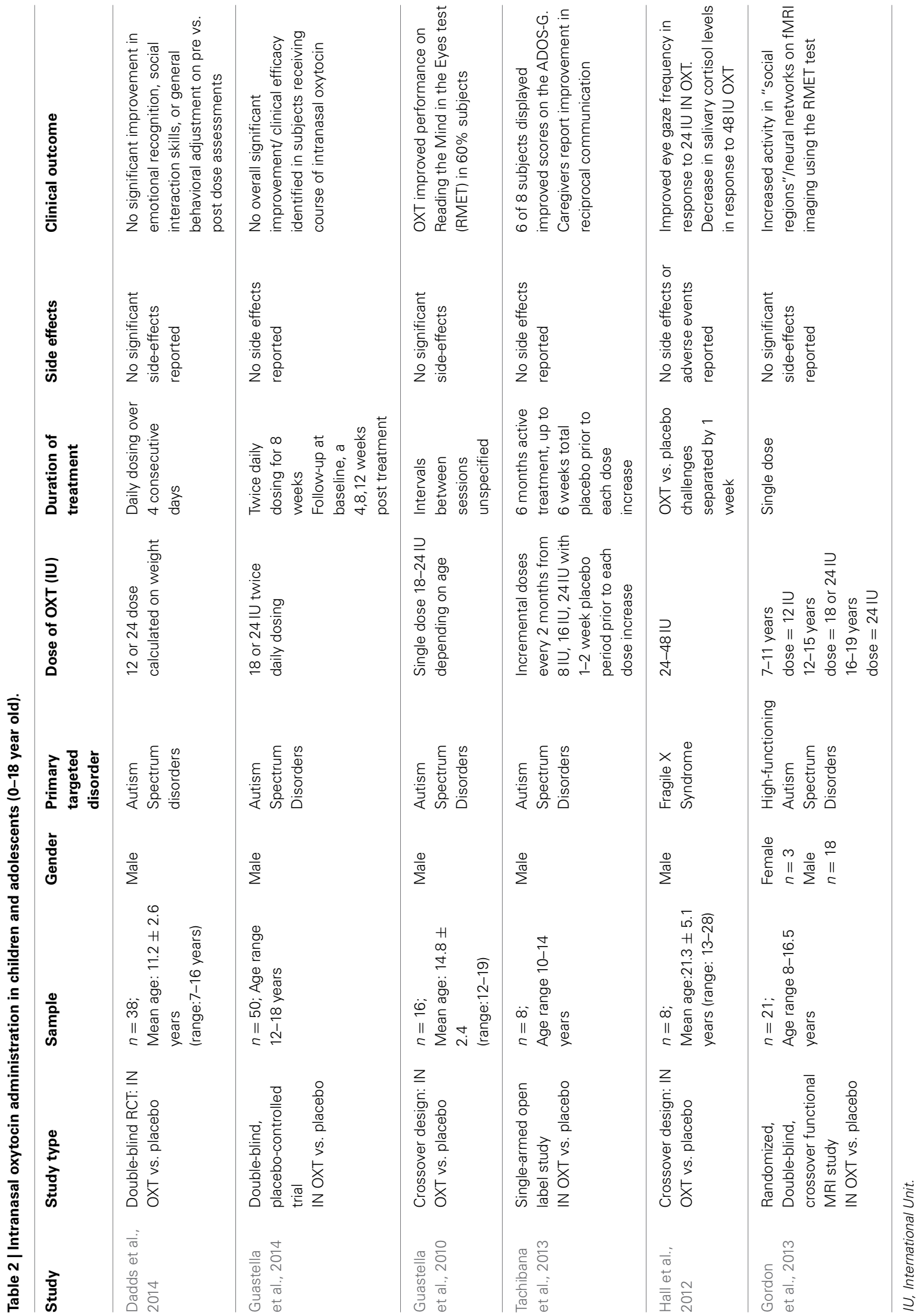


involving administration of intranasal oxytocin to both young males and females for various mental health disorders.

In recent reviews, authors have commented on "cascading effects" of intranasal oxytocin (Veening and Olivier, 2013) whereby administration of intranasal oxytocin also stimulates release of peripherally acting oxytocin (Veening and Olivier, 2013). One rat study did report increased basal levels of oxytocin after long-term administration during adolescence (Suraev et al., 2014); the opposite could be possible as well-depending on the age of exposure. Potentially detrimental effects of inadvertently raising systemic levels of oxytocin remain unclear both in the adult and pediatric population, and would require further investigation from a safety perspective.

\section{RISKS OF OXYTOCIN IN CHILDREN WITH SEVERE PSYCHOPATHOLOGY AND EARLY LIFE TRAUMA}

As outlined, the oxytocin neuropeptide system in humans remains in an evolutionary state with ongoing plasticity throughout infancy, early childhood, and during adolescence (BuismanPijlman et al., 2014b).

Those impacted by significant early life trauma are more likely to display a heightened degree of emotional dysregulation (Antoniadis et al., 2012). It is well-recognized in psychiatric practice that emotional dysregulation, impulsivity, and relationship instability are clinical features of psychopathology in Borderline Personality Disorder that are likely to be in an evolutionary state during adolescence (Barnow et al., 2012). The role of oxytocin in modulating the relational capacities in children and adolescents has been considered extensively in the literature, particular in relation to attachment disorders, anxiety and autism-spectrum disorders (Meyer-Lindenberg et al., 2011; Frijling et al., 2014).

The effect of intranasal oxytocin on these subjects might be difficult to predict. In addition, there will need to be a heightened awareness of the interconnectivity of for example the dopaminergic, serotonergic, and noradrenergic systems (Tops et al., 2014), and potential effects this poses, with oxytocin administration. In a clinical subgroup already plagued by attachment and relational difficulties, further concern is warranted given the risk of oxytocin administration leading to long-lasting behavioral and social, and neuroendocrine consequences (Miller, 2013).

The current research in fact suggests that oxytocin administration (intranasally) in the clinical settings for autism, social anxiety disorder, post-natal depression, obsessive-compulsive disorder, schizophrenia, borderline personality disorder, and posttraumatic stress disorder, yield minimal improvement in emotion recognition and interpersonal trust. In the context of developmental trauma, positive effects (behaviorally or neurobiologically) were in fact lowered or absent (Bakermans-Kranenburg and Van IJzendoorn, 2013). It may be more feasible to identify improvements in disorders that have a homogenous etiology and little comorbidity.

\section{DISCUSSION}

An evolving wealth of animal and human data relating to the neuro-modulatory role of the endogenous oxytocin system, its interactions with other neural pathways, and its implications on behaviors from a neuroscience perspective is expanding at an exponential rate (Tops et al., 2014). There is evidence highlighting (1) the importance of oxytocin in attachment (Love, 2014) and (2)the factors that may lead to individual variations in the endogenous oxytocin system including early life trauma and environmental factors (Buisman-Pijlman et al., 2014b). This highlights further potential research questions based around whether attachment difficulties may be in part modifiable not only through psycho-education and implementation of behavioral strategies, but through implementation of pharmacotherapies for high-risk populations.

Furthermore the benefits of intranasal oxytocin administration in the adult population are now also becoming clearer, and the potential clinical applications more far-reaching. Researchers are now investigating the potential use of intranasal oxytocin in the treatment of depression as an adjunct to selective serotonin reuptake inhibitors (Emiliano et al., 2007), anxiety (Guastella et al., 2009), and more recently, anorexia nervosa (Kim et al., 2014) with promising preliminary results findings. This data would be of high clinical relevance if translated to trials targeting the adolescent population in which affective, anxiety and eating disorders are also prevalent. There are limited animal and human studies reflecting consistent results to date in pediatric populations, with the vast majority focusing on the use of intranasal oxytocin in modifying behaviors related to ASDs. There are fundamental considerations relating to neuroplasticity, neuroadaptation and the impact of significant psychopathology that remain inadequately explored, and will likely inform further discussions around the safety of intranasal oxytocin as a therapeutic intervention. Based on our knowledge of the context-dependent nature (Guastella and Macleod, 2012), and plasticity of oxytocin system (Buisman-Pijlman et al., 2014b), animal and human studies are challenging to translate to pediatric populations. In addition, further questions relating to sensitive and specific methods of measuring outcomes in potential trials need consideration. Addressing these issues could lead to a broader wealth of research relating to oxytocin in pediatric populations, findings of which would likely continue to inform the way in which therapeutic interventions in the child and adolescent population are considered in the future.

\section{REFERENCES}

Ansseau, M., Legros, J. J., Mormont, C., Cerfontaine, J. L., Papart, P., Geenen, V., et al. (1987). Intranasal oxytocin in obsessive-compulsive disorder. Psychoneuroendocrinology 12, 231-236. doi: 10.1016/0306-4530(87)90009-6

Antoniadis, D., Samakouri, M., and Livaditis, M. (2012). The association of bipolar spectrum disorders and borderline personality disorder. Psychiatr. Q. 83, 449-465. doi: 10.1007/s11126-012-9214-6

Bakermans-Kranenburg, M. J., and Van IJzendoorn, M. H. (2013). Sniffing around oxytocin: review and meta-analyses of trials in healthy and clinical groups with implications for pharmacotherapy. Transl. Psychiatry 3, e258. doi: 10.1038/tp.2013.34

Bales, K. L., and Perkeybile, A. M. (2012). Developmental experiences and the oxytocin receptor system. Horm. Behav. 61, 313-319. doi: 10.1016/j.yhbeh.2011.12.013

Bales, K. L., Perkeybile, A. M., Conley, O. G., Lee, M. H., Guoynes, C. D., Downing, G. M., et al. (2013). Chronic intranasal oxytocin causes long-term impairments in partner preference formation in male prairie voles. Biol. Psychiatry 74, 180-188. doi: 10.1016/j.biopsych.2012.08.025

Bales, K. L., Pfeifer, L. A., and Carter, C. S. (2004). Sex differences and developmental effects of manipulations of oxytocin on alloparenting and anxiety in prairie voles. Dev. Psychobiol. 44, 123-131. doi: 10.1002/dev.10165 
Bales, K. L., Van Westerhuyzen, J. A., Lewis-Reese, A. D., Grotte, N. D., Lanter, J. A., and Carter, C. S. (2007). Oxytocin has dose-dependent developmental effects on pair-bonding and alloparental care in female prairie voles. Horm. Behav. 52, 274-279. doi: 10.1016/j.yhbeh.2007.05.004

Barnow, S., Limberg, A., Stopsack, M., Spitzer, C., Grabe, H. J., Freyberger, H. J., et al. (2012). Dissociation and emotion regulation in borderline personality disorder. Psychol. Med. 42, 783-794. doi: 10.1017/S0033291711001917

Baumgartner, T., Heinrichs, M., Vonlanthen, A., Fischbacher, U., and Fehr, E. (2008). Oxytocin shapes the neural circuitry of trust and trust adaptation in humans. Neuron 58, 639-650. doi: 10.1016/j.neuron.2008.04.009

Beetz, A., Uvnas-Moberg, K., Julius, H., and Kotrschal, K. (2012). Psychosocial and psychophysiological effects of human-animal interactions: the possible role of oxytocin. Front. Psychol. 3:234. doi: 10.3389/fpsyg.2012.00234

Bowen, M. T., Carson, D. S., Spiro, A., Arnold, J. C., and McGregor, I. S. (2011). Adolescent oxytocin exposure causes persistent reductions in anxiety and alcohol consumption and enhances sociability in rats. PLOS ONE 6:e27237. doi: 10.1371/journal.pone.0027237

Bowlby, J. (1960). Separation anxiety. Int. J. Psychoanal. 41, 89-113.

Broadbear, J. H., Kabel, D., Tracy, L., and Mak, P. (2014). Oxytocinergic regulation of endogenous as well as drug-induced mood. Pharmacol. Biochem. Behav. 119, 61-71. doi: 10.1016/j.pbb.2013.07.002

Buisman-Pijlman, F. T., Broadbear, J. H., and Sarnyai, Z. (2014a). Guest editorial. The role of oxytocin in positive affect and drug-related reward. Pharmacol. Biochem. Behav. 119, 1-2. doi: 10.1016/j.pbb.2013.11.023

Buisman-Pijlman, F. T., Sumracki, N. M., Gordon, J. J., Hull, P. R., Carter, C. S., and Tops, M. (2014b). Individual differences underlying susceptibility to addiction: role for the endogenous oxytocin system. Pharmacol. Biochem. Behav. 119, 22-38. doi: 10.1016/j.pbb.2013.09.005

Campbell, D. B., Datta, D., Jones, S. T., Batey Lee, E., Sutcliffe, J. S., Hammock, E. A., et al. (2011). Association of oxytocin receptor (OXTR) gene variants with multiple phenotype domains of autism spectrum disorder. J. Neurodev. Disord. 3, 101-112. doi: 10.1007/s11689-010-9071-2

Churchland, P. S., and Winkielman, P. (2012). Modulating social behavior with oxytocin: how does it work? What does it mean? Horm. Behav. 61, 392-399. doi: 10.1016/j.yhbeh.2011.12.003

Cohen, J., Danezis, J., and Burnhill, M. S. (1962). Response of the gravid uterus at term to intranasal oxytocin as determined by intra-amniotic fluid pressure recordings. Am. J. Obstet. Gynecol. 83, 774-777.

Dadds, M. R., Macdonald, E., Cauchi, A., Williams, K., Levy, F., and Brennan, J. (2014). Nasal oxytocin for social deficits in childhood autism: a randomized controlled trial. J. Autism Dev. Disord. 44, 521-531. doi: 10.1007/s10803-0131899-3

Den Boer, J. A., and Westenberg, H. G. (1992). Oxytocin in obsessive compulsive disorder. Peptides 13, 1083-1085. doi: 10.1016/0196-9781(92)90010-Z

Dolen, G., Darvishzadeh, A., Huang, K. W., and Malenka, R. C. (2013). Social reward requires coordinated activity of nucleus accumbens oxytocin and serotonin. Nature 501, 179-184. doi: 10.1038/nature12518

Emiliano, A. B., Cruz, T., Pannoni, V., and Fudge, J. L. (2007). The interface of oxytocin-labeled cells and serotonin transporter-containing fibers in the primate hypothalamus: a substrate for SSRI's therapeutic effects? Neuropsychopharmacology 32, 977-988. doi: 10.1038/sj.npp.1301206

Feldman, R. (2012). Oxytocin and social affiliation in humans. Horm. Behav. 61, 380-391. doi: 10.1016/j.yhbeh.2012.01.008

Frijling, J. L., Van Zuiden, M., Koch, S. B., Nawijn, L., Goslings, J. C., Luitse, J. S., et al. (2014). Efficacy of oxytocin administration early after psychotrauma in preventing the development of PTSD: study protocol of a randomized controlled trial. BMC Psychiatry 14:92. doi: 10.1186/1471244X-14-92

Gordon, I., Vander Wyk, B. C., Bennett, R. H., Cordeaux, C., Lucas, M. V., Eilbott, J. A., et al. (2013). Oxytocin enhances brain function in children with autism. Proc. Natl. Acad. Sci. U.S.A. 110, 20953-20958. doi: 10.1073/pnas.1312857110

Guastella, A. J., Einfeld, S. L., Gray, K. M., Rinehart, N. J., Tonge, B. J., Lambert, T. J., et al. (2010). Intranasal oxytocin improves emotion recognition for youth with autism spectrum disorders. Biol. Psychiatry 67, 692-694. doi: 10.1016/j.biopsych.2009.09.020

Guastella, A. J., Gray, K. M., Rinehart, N. J., Alvares, G. A., Tonge, B. J., Hickie, I. B., et al. (2014). The effects of a course of intranasal oxytocin on social behaviors in youth diagnosed with autism spectrum disorders: a randomized controlled trial. J. Child Psychol. Psychiatry. doi: 10.1111/jcpp.12305. [Epub ahead of print].
Guastella, A. J., Howard, A. L., Dadds, M. R., Mitchell, P., and Carson, D. S. (2009). A randomised control trial of intranasal oxytocin as an adjunct to exposure therapy for social anxiety disorder. J. Psychoneuroendocrinology 34, 917-923. doi: 10.1016/j.psyneuen.2009.01.005

Guastella, A. J., and Macleod, C. (2012). A critical review of the influence of oxytocin nasal spray on social cognition in humans: evidence and future directions. Horm. Behav. 61, 410-418. doi: 10.1016/j.yhbeh.2012.01.002

Hall, S. S., Lightbody, A. A., McCarthy, B. E., Parker, K. J., and Reiss, A. L. (2012). Effects of intranasal oxytocin on social anxiety in males with fragile $\mathrm{X}$ syndrome. Psychoneuroendocrinology 37, 509-518. doi: 10.1016/j.psyneuen.2011.07.020

Heim, C., Young, L. J., Newport, D. J., Mletzko, T., Miller, A. H., and Nemeroff, C. B. (2009). Lower CSF oxytocin concentrations in women with a history of childhood abuse. Mol. Psychiatry 14, 954-958. doi: 10.1038/mp.2008.112

Heinrichs, M., Baumgartner, T., Kirschbaum, C., and Ehlert, U. (2003). Social support and oxytocin interact to suppress cortisol and subjective responses to psychosocial stress. Biol. Psychiatry 54, 1389-1398. doi: 10.1016/S00063223(03)00465-7

Hendricks, C. H., and Pose, S. V. (1961). Intranasal oxytocin in obstetrics. JAMA 175, 384-387. doi: 10.1001/jama.1961.03040050040009

Johns, J. M., Noonan, L. R., Zimmerman, L. I., McMillen, B. A., Means, L. W., Walker, C. H., et al. (1998). Chronic cocaine treatment alters social/aggressive behavior in Sprague-Dawley rat dams and in their prenatally exposed offspring. Ann. N.Y. Acad. Sci. 846, 399-404. doi: 10.1111/j.1749-6632.1998. tb09765.x

Kim, Y. R., Oh, S. M., Corfield, F., Jeong, D. W., Jang, E. Y., and Treasure, J. (2014). Intranasal oxytocin lessens the attentional bias to adult negative faces: a double blind within-subject experiment. Psychiatry Investig. 11, 160-166. doi: 10.4306/pi.2014.11.2.160

Knobloch, H. S., Charlet, A., Hoffmann, L. C., Eliava, M., Khrulev, S., Cetin, A. H., et al. (2012). Evoked axonal oxytocin release in the central amygdala attenuates fear response. Neuron 73, 553-566. doi: 10.1016/j.neuron.2011.11.030

Kovacs, G. L., Sarnyai, Z., and Szabo, G. (1998). Oxytocin and addiction: a review. Psychoneuroendocrinology 23, 945-962. doi: 10.1016/S0306-4530(98) 00064-X

Lee, H. J., Macbeth, A. H., Pagani, J. H., and Young, W. S. 3rd. (2009). Oxytocin: the great facilitator of life. Prog. Neurobiol. 88, 127-151. doi: 10.1016/j.pneurobio.2009.04.001

Love, T. M. (2014). Oxytocin, motivation and the role of dopamine. Pharmacol. Biochem. Behav. 119, 49-60. doi: 10.1016/j.pbb.2013.06.011

McGregor, I. S., and Bowen, M. T. (2012). Breaking the loop: oxytocin as a potential treatment for drug addiction. Horm. Behav. 61, 331-339. doi: 10.1016/j.yhbeh.2011.12.001

Meyer-Lindenberg, A., Domes, G., Kirsch, P., and Heinrichs, M. (2011). Oxytocin and vasopressin in the human brain: social neuropeptides for translational medicine. Nat. Rev. Neurosci. 12, 524-538. doi: 10.1038/nrn3044

Miller, G. (2013). Neuroscience. The promise and perils of oxytocin. Science 339, 267-269. doi: 10.1126/science.339.6117.267

Miller, M., Bales, K. L., Taylor, S. L., Yoon, J., Hostetler, C. M., Carter, C. S., et al. (2013). Oxytocin and vasopressin in children and adolescents with autism spectrum disorders: sex differences and associations with symptoms. Autism Res. 6, 91-102. doi: 10.1002/aur.1270

Olff, M. (2012). Bonding after trauma: on the role of social support and the oxytocin system in traumatic stress. Eur. J. Psychotraumatol. 3:18597. doi: 10.3402/ejpt.v3i0.18597

Opacka-Juffry, J., and Mohiyeddini, C. (2012). Experience of stress in childhood negatively correlates with plasma oxytocin concentration in adult men. Stress 15, 1-10. doi: 10.3109/10253890.2011.560309

Oreland, S., Gustafsson-Ericson, L., and Nylander, I. (2010). Short- and longterm consequences of different early environmental conditions on central immunoreactive oxytocin and arginine vasopressin levels in male rats. Neuropeptides 44, 391-398. doi: 10.1016/j.npep.2010.06.001

Parker, K. J., Buckmaster, C. L., Schatzberg, A. F., and Lyons, D. M. (2005). Intranasal oxytocin administration attenuates the ACTH stress response in monkeys. Psychoneuroendocrinology 30, 924-929. doi: 10.1016/j.psyneuen.2005.04.002

Pedersen, C. A., Smedley, K. L., Leserman, J., Jarskog, L. F., Rau, S. W., KampovPolevoi, A., et al. (2013). Intranasal oxytocin blocks alcohol withdrawal in human subjects. Alcohol. Clin. Exp. Res. 37, 484-489. doi: 10.1111/j.15300277.2012.01958.x 
Pitman, R. K., Orr, S. P., and Lasko, N. B. (1993). Effects of intranasal vasopressin and oxytocin on physiologic responding during personal combat imagery in Vietnam veterans with posttraumatic stress disorder. Psychiatry Res. 48, 107-117. doi: 10.1016/0165-1781(93)90035-F

Preti, A., Melis, M., Siddi, S., Vellante, M., Doneddu, G., and Fadda, R. (2014). Oxytocin and autism: a systematic review of randomized controlled trials. J. Child Adolesc. Psychopharmacol. 24, 54-68. doi: 10.1089/cap.2013.0040

Rault, J. L., Carter, C. S., Garner, J. P., Marchant-Forde, J. N., Richert, B. T., and Lay, D. C. Jr. (2013). Repeated intranasal oxytocin administration in early life dysregulates the HPA axis and alters social behavior. Physiol. Behav. 112-113, 40-48. doi: 10.1016/j.physbeh.2013.02.007

Rilling, J. K., Demarco, A. C., Hackett, P. D., Thompson, R., Ditzen, B., Patel, R., et al. (2012). Effects of intranasal oxytocin and vasopressin on cooperative behavior and associated brain activity in men. Psychoneuroendocrinology 37, 447-461. doi: 10.1016/j.psyneuen.2011.07.013

Sarnyai, Z., and Kovacs, G. L. (2014). Oxytocin in learning and addiction: from early discoveries to the present. Pharmacol. Biochem. Behav. 119, 3-9. doi: 10.1016/j.pbb.2013.11.019

Simpson, E. A., Sclafani, V., Paukner, A., Hamel, A. F., Novak, M. A., Meyer, J. S., et al. (2014). Inhaled oxytocin increases positive social behaviors in newborn macaques. Proc. Natl. Acad. Sci. U.S.A. 111, 6922-6927. doi: $10.1073 /$ pnas. 1402471111

Suraev, A. S., Bowen, M. T., Ali, S. O., Hicks, C., Ramos, L., and McGregor, I. S. (2014). Adolescent exposure to oxytocin, but not the selective oxytocin receptor agonist TGOT, increases social behavior and plasma oxytocin in adulthood. Horm. Behav. 65, 488-496. doi: 10.1016/j.yhbeh.2014.03.002

Tachibana, M., Kagitani-Shimono, K., Mohri, I., Yamamoto, T., Sanefuji, W., Nakamura, A., et al. (2013). Long-term administration of intranasal oxytocin is a safe and promising therapy for early adolescent boys with autism spectrum disorders. J. Child Adolesc. Psychopharmacol. 23, 123-127. doi: 10.1089/cap. 2012.0048
Tops, M., Koole, S. L., IJzerman, H., and Buisman-Pijlman, F. T. (2014). Why social attachment and oxytocin protect against addiction and stress: insights from the dynamics between ventral and dorsal corticostriatal systems. Pharmacol. Biochem. Behav. 119, 39-48. doi: 10.1016/j.pbb.2013.07.015

Veening, J. G., and Olivier, B. (2013). Intranasal administration of oxytocin: behavioral and clinical effects, a review. Neurosci. Biobehav. Rev. 37, 1445-1465. doi: 10.1016/j.neubiorev.2013.04.012

Williams, S. K., and Johns, J. M. (2014). Prenatal and gestational cocaine exposure: effects on the oxytocin system and social behavior with implications for addiction. Pharmacol. Biochem. Behav. 119, 10-21. doi: 10.1016/j.pbb.2013.07.004

Zanos, P., Wright, S. R., Georgiou, P., Yoo, J. H., Ledent, C., Hourani, S. M., et al. (2014). Chronic methamphetamine treatment induces oxytocin receptor up-regulation in the amygdala and hypothalamus via an adenosine A2A receptor-independent mechanism. Pharmacol. Biochem. Behav. 119, 72-79. doi: 10.1016/j.pbb.2013.05.009

Conflict of Interest Statement: The authors declare that the research was conducted in the absence of any commercial or financial relationships that could be construed as a potential conflict of interest.

Received: 25 June 2014; accepted: 29 September 2014; published online: 16 October 2014.

Citation: Taylor AE, Lee H and Buisman-Pijlman FTA (2014) Oxytocin treatment in pediatric populations. Front. Behav. Neurosci. 8:360. doi: 10.3389/fnbeh.2014.00360 This article was submitted to the journal Frontiers in Behavioral Neuroscience.

Copyright (C) 2014 Taylor, Lee and Buisman-Pijlman. This is an open-access article distributed under the terms of the Creative Commons Attribution License (CC BY). The use, distribution or reproduction in other forums is permitted, provided the original author(s) or licensor are credited and that the original publication in this journal is cited, in accordance with accepted academic practice. No use, distribution or reproduction is permitted which does not comply with these terms. 